\title{
DECISION SUPPORT FOR CONFIGURATION SYSTEMS OF INDUSTRIALIZED CONSTRUCTIONS
}

\author{
Tamás Rácz* and Thomas Olofsson \\ Department of Civil Mining and Environmental Engineering, Luleå University of Technology, Luleå, Sweden \\ *Corresponding author (tamas.racz@ltu.se)
}

\begin{abstract}
The limitation in the design space of industrialized housing opens up possibilities for the creation of customer focused configuration systems and for increased level of design automation. The creation and performance analysis of design variants can be made quicker and be of better quality with the help of such configuration systems. Still the selection of the optimal alternative from the increasing number of design variants can be difficult when several interrelated and many times contradicting design parameters are available. Therefore the application of decision-making methods in helping the decision process is desirable. This paper is demonstrating the implementation of the decision-making framework called Smart DMF for an intended experimental configuration system. The product of the implementation is a software module supplementing a previously developed automated analysis module with the addition of new aspects like cost and user satisfaction factors. With the help of the Smart DMF module the otherwise complex picture of comparing design variants can be simplified; each variant can have a cumulative overall rating which helps to make the decisions sounder and less affected by the biases of human decisions. Moreover, we will see that after the capture of the decision making criteria and parameters the module can be used to find optimal design variants automatically.
\end{abstract}

Keywords: Industrialized Construction, Configuration System, Automation, Decision-making

\section{INTRODUCTION}

Industrialized construction systems such as Moderna HUS [1] from Skanska AB [2] are serving the ambitions of being faster, cheaper and of more stable quality to build [3][4], but have constrained design flexibility. In the Moderna HUS approach, which is dealing with multi-family, multistorey residential buildings, the geometry of the building, the exact layout of the flats, the employed construction technology, material and installations are all pre-defined during the development process of the products and only a limited set of parameters left open for user choice. Apart from the location and orientation parameters of the building the client can only select the number of stories, quality of finishes or appliances and the desired energy efficiency package of the design. These constraints open up possibilities for high degree of automations in all aspects of the construction process from client interactions to assembly. A previous article of the authors introduced automated performance analysis for a selected Moderna
HUS product that is capable of delivering basic performance values, which can be used in decision-making processes [5]. However it was noted that even with few number open design parameters the evaluation of the numerous possible parameter combinations - called variants -, practically the multi-parameter optimization of the building could be difficult. Therefore this paper presents an experimental development of a decisionmaking support module based on the Smart DMF decisionmaking framework [6][7], which combines Key Performance Indicators (KPI) calculated through utility functions reflecting user preferences and uses the renowned criteria comparison and ranking method of Saaty's Analytical Hierarchy Process [8].

\section{PERFORMANCE ANALYSIS MODULE WITH DECISION SUPPORT}

The performance analysis module presented in [5] is made to be part of a complete configuration system. It analyses 
energy performance with VIPCore [9] and uses an Excel based user interface. It is capable of analyzing several configuration variants almost in real-time. The previous experiment's functionalities are preserved and supplemented with simple cost calculation and the Smart DMF evaluation framework. The combined list of input for existing and new functionalities is presented below.

Location of building: It identifies the intended site of the building, which controls climate and cost parameters. The climate conditions are actual meteorological observations provided by NOAA-CIRES Climate Diagnostics Center [10].

Orientation of building: The orientation allows adjustment to panoramic needs and affects solar energy intake.

Number of floors: The selected Moderna HUS product allows 3 to 8 levels buildings.

Surrounding geography: Defines the horizontal angles of obstacles that shade the building from solar radiation. It has one horizontal angle indicating the lowest visible sun elevation for each of the eight principal geographic directions.

Cost components: Consists of site, building and building storey related cost components defined for each location.

Utility functions: These are used to calculate Key Performance Indicators (KPI) from input parameters and calculated building performance results. Each function transforms corresponding parameter or result into dimensionless $0-100 \%$ value, reflecting client acceptance.

Criteria comparison: Employs Saaty's criteria prioritization [8] by comparing the relative importance of the different parameters and performance results commonly called criteria - and calculating the priority ratio of each criterion.

The module produces the following results:

Heating requirements result: The VIPCore calculated annual heating requirement of the building per $\mathrm{m}^{2}$ of living area.

Construction cost result: The result of the module's simplified construction cost calculation based on the cost components input and the number of floors.

Rating of variants result: This is the actual rating calculated by the Smart DMF method, combining utility functions' KPI and priority ratio of each criterion. Equation 1 shows the generic formula of calculating variant ratings.

$$
\begin{aligned}
& R=\sum_{i=1}^{n_{c}} u_{i}\left(P_{i}\right) \cdot r_{i} \\
& R: \text { rating of variant } \\
& u_{i}(x): \text { utility function of criterion } i \\
& P_{i}: \text { performance value of the criterion } i \\
& u(P): \text { KPI value of the criterion } \\
& r_{i}: \text { priority ratio of the criterion } i \\
& n_{c}: \text { number of criteria } \\
& \text { Equation } 1: \text { Calculation of variants' rating }
\end{aligned}
$$

Although the above parameters are not representing directly articulated requirements in practice they are selected to satisfy the needs of the experiment and to represent possible real life situations or they reflect intrinsic attribute or condition. The number of floors is an intrinsic attribute of the underlying trial example. The location of building and the orientation of building are natural positioning attributes. The surrounding geography inputs are determined by the way the chosen energy analysis tool of VIPCore handles this aspect. The utility functions and the criteria comparison are the necessary input for the Smart DMF framework while ranking of variants result is its product. The cost components input and the way the construction cost result is calculated are employing highly simplified estimation. The construction cost is calculated as seen in Equation 2.

$$
\begin{aligned}
& C=\frac{C_{S}+C_{B}+C_{L} \cdot\left(n_{L}-1\right)}{A_{L} \cdot n_{L}} \\
& C: \text { cost of building } / \mathrm{m}^{2} \text { living area } \\
& C_{S}: \text { site component of cost } \\
& C_{B}: \text { building component of cost } \\
& C_{L}: \text { level component of cost } \\
& n_{L}: \text { number of levels in the building } \\
& A_{L}: \text { living area on one level }
\end{aligned}
$$

Equation 2: Simplified cost calculation of the experiment.

From among all the possible energy performance results the heating requirements result is selected as representative of the long-term performance indicator in mid and high 
latitude locations where Moderna HUS is introduced. The whole calculation is based on the Moderna HUS product called Grönskär. The input dataset of the energy performance calculation is provided by the responsible energy expert of the Skanska AB from operational project files catalog of the Grönskär product.

\section{EXAMPLES OF USE}

We demonstrate the use of the module through two hypothetical cases of clients who wishes to build multifamily house based on the Moderna HUS approach. One of the clients wishes to build flats for price sensitive customers while the other wishes to build for customers demand high living standard and better energy efficiency but has lower budgetary constraints. Their criteria preference matrix representing the relative priorities can be seen in Figure 1. Both clients have potential customers in Sweden and in the UK - where Moderna HUS is introduced so far - and wish to select one location from a shared pool of 6 . The sites have the same geographical, climate and cost conditions for the two clients. The geographical conditions are arbitrarily constructed for the experiment (Figure 4). Because of the higher standard requirements of the Client no. 2 the construction costs for this client are higher reflecting the higher standard of interior and façade components. The cost components are arbitrary but are producing costs being close to average 2009 market values taken from statistical databases [11][12], with the assumption that 5 level Grönskär buildings could be considered as typical (Figure 2).

The utility functions evaluating the performance of the input parameters and the result values have higher sensitivity towards expensive locations and construction costs but lower towards number of levels for Client 1 . Client 2 has preference in metropolitan locations and prefers fewer floor levels. For Client 1, who has fewer objections against high-rise buildings, we modeled exceptional conditions for Göteborg where the location gives picturesque view for high buildings. See the utility function details in Figure 3. The perception of optimal orientation is the same for the two clients across the locations.

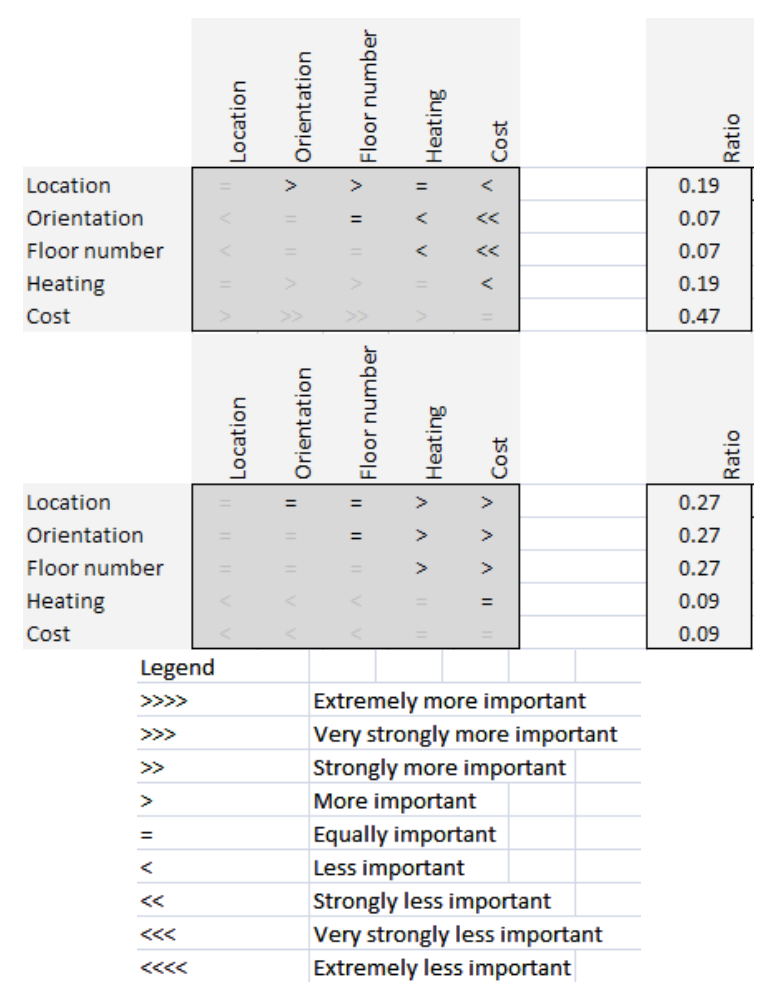

Figure 1: Criteria preference of Client 1 (top) and Client 2 (middle) with legend (bottom)

\begin{tabular}{|c|c|c|c|c|c|c|c|}
\hline Location & $\begin{array}{l}\begin{array}{l}\text { Site } \\
\mathrm{x} 1000 \mathrm{EUR}\end{array} \\
\end{array}$ & $\begin{array}{l}\text { Building } \\
\text { x1000EUR }\end{array}$ & $\begin{array}{l}\text { Floor } \\
\times 1000 \text { EUR }\end{array}$ & Location & $\begin{array}{l}\text { Site } \\
\text { x1000EUR }\end{array}$ & $\begin{array}{l}\text { Building } \\
\text { x1000EUR }\end{array}$ & $\begin{array}{l}\text { Floor } \\
\times 1000 \text { EUR }\end{array}$ \\
\hline Stockholm & 910 & 1100 & 780 & Stockholm & 910 & 1300 & 880 \\
\hline Göteborg & 825 & 1000 & 710 & Göteborg & 825 & 1200 & 810 \\
\hline Malmö & 910 & 1150 & 800 & Malmö & 910 & 1350 & 900 \\
\hline Luleå & 350 & 900 & 630 & Luleå & 350 & 1100 & 730 \\
\hline London & 1650 & 1300 & 920 & London & 1650 & 1500 & 1020 \\
\hline Brighton & 360 & 1100 & 770 & Brighton & 360 & 1300 & 870 \\
\hline
\end{tabular}

Figure 2: Simplified cost calculation components for Client 1 (left) and Client 2 (right)

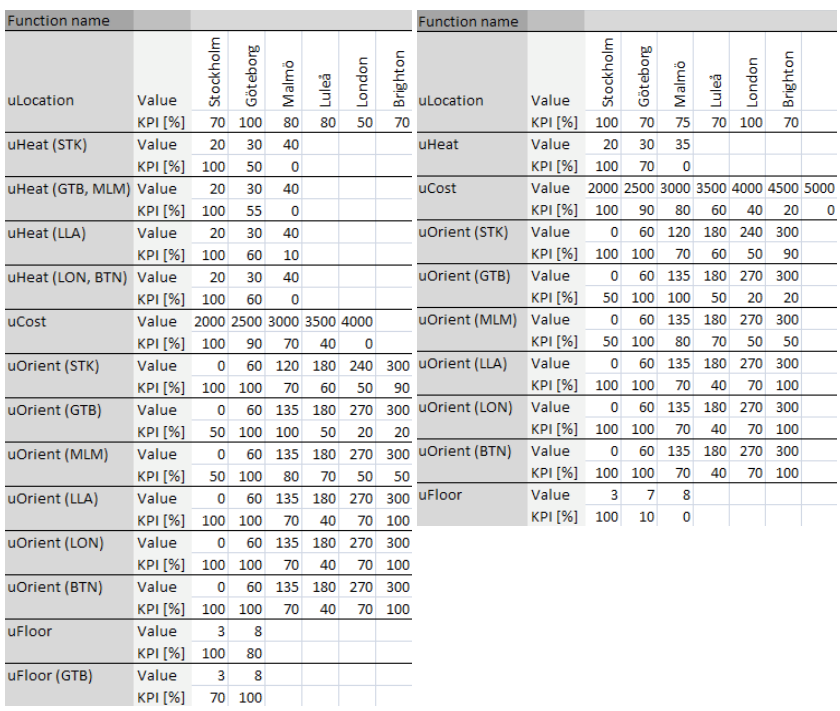

Figure 3: Utility functions for Client 1 (left) and Client 2 (right). 
Please note that for a certain criterion there might be several utility functions, each belonging to certain values of other criteria. For example the utility function assessing the orientation preferences depends on the location. In each location the orientation and consequently the view from the building could have different priorities. Since Client 1 is more sensitive towards the cost of heating there are different utility function of heating for different locations, representing the variation in energy prices across the locations. Client 2 has the same heating utility function for all locations but has a lower limit for the allowed maximum consumption.

The utility functions should assess underlying concepts clearly, i.e. the cost of energy should be considered only in the utility functions correspond to heating requirement and not in the utility function for the location, which considers living cost at the specific location.

For both clients we intend to select the optimal combination of location, orientation and floor number values so that it could satisfy their customer groups the most, based on the captured preferences. Therefore for each location we analyze a complete turn of orientation of the building in 30 degrees steps with all possible number of floor levels. This way we will have $6 \times 12 \times 6=432$ variants to choose from for each client. The calculation result of heating and cost together with location, orientation and number of floors input will be evaluated through the Smart DMF into a single rating value, which represents the combined acceptance of all variants on a 0 $100 \%$ scale.

Executing the analysis for all of the variants takes approximately 3 minutes for each client and produces the result presented in Figure 4. We can see that the location of Göteborg and the orientation of 60 degrees with 8 levels is the optimal variant based on the preferences of Client 1 . As of Client 2 the fairly expensive Stockholm location is the optimal one with the least possible level number of 3 and orientation of 30 degrees.

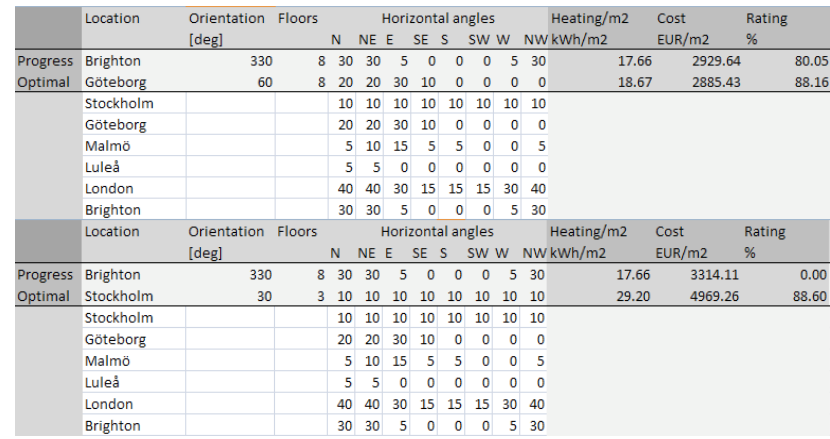

Figure 4: Optimal variant for Client 1 (top) and for Client 2 (bottom) with geography input of the locations (horizontal angles)

\section{DISCUSSION AND CONCLUSIONS}

The operation of the module is quick. It can provide simple benchmark result for variants under evaluation almost realtime, making it useful during negotiations. The evaluation is systematic: once the preferences are registered it lacks of human bias and it produces the same result for the same input regardless of time, situation and involved persons. Although, the Smart DMF method requires considerable amount of preparation even for limited number of parameters, the definition of utility functions and criteria preferences ensures the possibility of review and tracking of the decision making process. The reproducibility and transparency of the method is valuable when we need to reevaluate decision makings due to changing conditions: we might be certain that the results will reflect only changes in the formal input parameters and will not contain other, hidden ad-hoc human biases.

To ease the workload during the preparation of the demonstration scenario the possibility of automation for the generation of $2 \times 432$ variants was investigated. A simple variant creation functionality was invented which eventually transformed into a basic optimal variant seeking automation: after specifying the necessary inputs with desired range of open parameters - in our case the 6 locations with dependent data, 0-360 degrees of orientation in 30 degrees steps and 3-8 number of levels - the module generates and calculates all possible parameter combinations autonomously and present single optimal result in the end. Figure 4 shows part of the optimization interface where the progress line shows the actual 
configuration being calculated while the optimal line shows the variant with the highest rating so far. Separate worksheet of the Excel interface shows all the variants that been analyzed so those can be reviewed (part of this sheet is shown in Figure 5). This way the decision-making procedure is further automated, eliminating manual generation of variants.

\begin{tabular}{|c|c|c|c|c|c|c|c|c|c|c|c|c|c|c|}
\hline \multicolumn{2}{|r|}{ Q10 } & \multicolumn{2}{|c|}{$-\left(0 \quad f_{x}\right.$} & \multicolumn{11}{|c|}{$=100^{*} 0.273+100^{*} 0.273+100^{*} 0.273+72.3984^{*} 0.091+1.2296^{*} 0.091$} \\
\hline$\overline{4}$ & A & B & c & E & $\mathrm{F}$ & G & $\mathrm{H}$ & & & $\mathrm{K}$ & M & P & Q & \\
\hline 2 & Location & ntation & Floors & & orizo & onta & I ang & gles & & & ing & Cost & Rating & \\
\hline 3 & & [de & $\mathrm{N}$ & NE E & & SE S & & sW W & & & $\mathrm{h} / \mathrm{m} 2$ & EUR/m2 & & Locatiol \\
\hline 8 & Stockholm & 0 & 710 & $\begin{array}{ll}010 \\
\end{array}$ & & 10 & 101 & 101 & 10 & 10 & 24.68 & $\begin{array}{l}3677.5 \\
\end{array}$ & 69.97 & 7 uLocatis \\
\hline 9 & Stockho & 0 & 810 & 010 & 10 & 10 & 101 & 101 & 10 & 10 & 30 & 3556.32 & 0.0 & \\
\hline 10 & Stockh & 30 & 310 & 010 & 10 & 10 & 101 & 101 & 10 & 10 & 20 & 4969.26 & 88.60 & 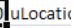 \\
\hline 11 & Stockho & 30 & 410 & 010 & 10 & 10 & 101 & 101 & 10 & 10 & 08 & $4404.3^{\circ}$ & 09 & \\
\hline 12 & Stockholm & 30 & 510 & 010 & 10 & 10 & 101 & 101 & 10 & 10 & 25.86 & 4065.19 & 80.52 & 24 \\
\hline & Q364 & & $f_{x}=$ & $=70 * 0$ & 0.273 & $3+10$ & $00 * 0$ & $.273+$ & +10 & $0 * 0$ & $273+100 * \mathrm{C}$ & ${ }^{*} 0.091+25^{*}$ & 0.091 & \\
\hline 4 & A & B & D & E & $\mathrm{F}$ & G & $\mathrm{H}$ & 1 & J & K & M & $\mathrm{P}$ & Q & \\
\hline 2 & Location & Orientation & Floors & & orizo & onta & I ang & gles & & & leating & Cost & Rating & Utility F \\
\hline 3 & & [deg] & $\mathrm{N}$ & NE E & & SE $S$ & & sW W & & & $\mathrm{Wh} / \mathrm{m} 2$ & EUR $/ \mathrm{m} 2$ & & Locatior \\
\hline 62 & London & 330 & 740 & 040 & 30 & 15 & 151 & 153 & 30 & 40 & 17.91 & 1 4521.09 & 68.17 & 7 uLocatic \\
\hline 63 & London & 330 & 840 & 040 & 30 & 15 & 151 & 153 & 30 & 40 & 7.86 & 54348.33 & 0.0 & catic \\
\hline 64 & Brighton & 0 & 330 & 030 & 5 & 0 & 0 & 0 & 5 & 30 & .75 & 4375 & \begin{tabular}{|l|} 
\\
\end{tabular} & IuLocatic \\
\hline 5 & on & 0 & 430 & 030 & 5 & 0 & 0 & 0 & & 30 & .71 & 1 3950.81 & & 9 uLocatic \\
\hline 66 & Brighton & 0 & 530 & 030 & 5 & 0 & 0 & 0 & & 30 & 17.69 & 9 3696.19 & 75.27 & 7 uLocatic \\
\hline
\end{tabular}

Figure 5: Calculation details of variants for Client 2

A detailed review of variant ranking uncovered the problem of non-transparency of very low performing low preference criteria. Figure 5 is showing parts of the calculation details of the optimal criteria for Client 2. The formula of the rating cell of the optimal variant in line 10 shows that the last component of the rating - which is the cost component - has $1.2 \%$ KPI value. Since other criteria performing well ( $72.4 \%$ or more) and since cost has low relative priority for Client 2 (0.091) this fact cannot be seen in the overall rating value of the variant alone. This phenomenon might have negative influence on the decision-making process by favoring an almost inadequate variant over more steadily performing ones. As a comparison, the variant in the line 364 of Figure 5 has nearly identical overall rating as the optimal one from line 10 but its criteria perform more balanced. Choosing the variant 364 might be a more natural choice considering that it has more attractive cost (its KPI is $25 \%$ in contrast of $1.2 \%$ ) and the important criteria (location, orientation and floor number, each with 0.273 priority ratio) have $100 \%$ KPI value. Rooting our decision solely on variant rating in this case ends up in investing in a completely different location than a more natural choice would suggest.
To overcome this phenomenon we propose two different kinds of possible approach. One is to invent more elaborate variant rating evaluation than the current one presented in Formula 1, which can indicate low performing criteria better. The other more simple is to introduce warning threshold for utility functions that alarms the users for criteria with KPI below threshold limit. It may be manifested in new column of the current Excel sheet based interface, alerting the user for variants with very low performing criteria, possibly indicating the amount the threshold been violation.

It should be noted that the method is intended to complement the decision process and not to be its sole technique. The method is only as reliable as its input. The formal capture of human intentions might not always be possible, or not to all of the details, and the prioritization of criteria might work differently from person to person, therefore additional considerations might be necessary. Still, the method offers transparent, stable and systematic way of considering user preferences in the evaluation of high number of decision variants.

\section{ACKNOWLEDGEMENTS}

We wish to express our appreciation towards Carl Jonsson and Björn Berggren from Skanska Teknik AB for all the information and data they provided concerning Moderna HUS and towards the Swedish Research Council Formas who sponsored our research (Dnr 244-2008-61).

\section{REFERENCES}

[1] Moderna HUS, Skanska, http://www.skanska.se /sv/Bygg-och-anlaggning/Byggkoncept/ModernaHus/, 2011 March

[2] Skanska AB, http://www.skanska.com/, 2011 March

[3] Jansson, G., "Industrialized Housing Design Efficiency", Licentiate Thesis, Luleå University of Technology, 2010

[4] Lessing, J., "Industrialised House-Building”, Licentiate Thesis, Luleå University of Technology, 2006

[5] Rácz, T., Rönneblad, A., Olofsson, T., "Energy Analysis Automation for Industrialzed Construction 
S6- 5

Processes", Proceedings of the 27th CIB W078 International Conference on Applications of IT in the AEC Industry, Cairo, 2010

[6] "A Smart Decision Making Framework for Building Information Models", http://www.inpro-project.eu/docs/ InPro_SmartDecisionMakingFrameworkBIM.pdf, 2011 March

[7] Schade, J., Olofsson T., Schreyer, M., 2011, "Decisionmaking in a model-based design process", Construction Management and Economics, Accepted for publication

[8] Saaty, T. L., "The Analytic Hierarchy Process", McGraw-Hill, New York, 1980

[9] VIPCore Calculation engine, http://www.strusoft.com/ index.php/en/modulesvip, 2011 March [10] NCEP Reanalysis data provided by NOAA-CIRES Climate Diagnostics Center, Boulder, Colorado, USA, http://www.esrl.noaa.gov, 2011 March

[11] Statistiska Centralbyrån (Statistics Sweden) Databases, http://www.ssd.scb.se, 2011 March

[12] Land Registry UK, http://www.landregistry.gov.uk, 2011 March 